\title{
Review and Prospect on Development of Urban Flood Warning System
}

\author{
Jianhua Li, Zhaosong Qu, Jun Zheng, Chong Jiang \\ Beijing Sinfotek Technology Company Limited
}

\begin{abstract}
An overall research and analysis on past development track was of great significance for the further development of new theories, models, technologies and applications of urban flood warning system. Thus, the paper comprehensively discussed the development track of urban flood warning system, especially its structures and supporting technologies. From the analysis, it could be seen clearly that demands and technologies were two major driving forces for the development of urban flood warning system in the era of big data. Moreover, it was illustrated that new demands and problems had to be faced, and discussed how to meet them in the future by integrating technologies of big data and cloud computing.
\end{abstract}

\section{Introduction}

The development of urban flood prevention is driven by demands of social development, development of monitoring and information technology. Annual rainfall in China is more than $1600 \mathrm{~mm}$ in southeast and only several in northwest[1]. Heavy rain in China usually occurs in the range of early July and late August. In recent years, however, there have been "early floods" in various places, and also been varying degrees of distress in north[2]. The problems of flood prevention are outstanding. Some small and medium-sized cities have also begun to appear the phenomenon of "sea city". The disaster of flood season has become a key factor that affects and restricts the development of cities in China. In the past, the center of gravity of urban flood prevention was mostly concentrated in rural areas, but now it's turning into the cities[3]. Flood control has become an important task in urban management.

With development and application of big data, cloud computing and internet of things, the urban flood warning system has changed a lot. It has been provided a new impetus to the development of urban flood prevention and early warning in terms of demand and technology[4].

In the paper, it was expounded the development of urban flood warning system in China, and new demands of urban flood prevention must be faced in a big data era. A summary of construction and detailed management of urban flood warning system at the present stage has been made, and a prospect has been made for urban flood prevention and early warning.

\section{A summary of the development of urban flood warning system}

For urban rainstorm warning management, many countries are focused on the combination of real-time monitoring and scientific early warning. For example, the government of America pay a great attention to early warning of meteorological disasters $[5,6]$. The earth meteorological satellite and numerical simulation technology have been used for monitoring and warning meteorological disasters for a long time in America[7]. According to meteorological information, meteorological satellites and other ways of observation should be strengthened to monitor severe weather and issue timely warning when disasters coming.

Compared with the developed countries in Europe and Amarica, the research and application of the early warning system in China started a little late. With increaseing in the scale of cities, disaster losses caused by extreme weather and awareness of disaster prevention have also been increased.

\subsection{Theoretical research stage}

Indicators and methods for flood assessment had been established by some Chinese experts on the basis of relationships between function of flood prevention science system, logical structure, hierarchy and subsystems[8]. The formation of floods is the result of the combination of hazards, disasters, environments and disaster mechanisms resulting from the process of surface change on the earth.

\subsection{Drawing flood risk maps and studying disasters}

In China, the study of flood risk maps is just at the beginning. Although the definition of flood risk is still controversial, it has been discussed as a single discipline by various disciplines. First, we have estimated the 
probability of different flood levels in rivers and compiled a number of special maps. Second, the flood water level was measured and the flood water level was estimated.Third, it studies the variation of flood in spatial and temporal changes of land use.Fourth,A flood risk model system was applied and proposed.Fifth, the disaster caused by floods has been measured by modern earth observation technology, which has played an important role in ensuring the formulation of emergency measures[9].

\subsection{New generation of information technology-aided decision-making}

As early as 1992, the "National Flood Control and Dispatching System" sponsored by the office of National Defense Command was prototype of national flood management decision support system. It includes computer local area network, prototype flood control comprehensive database, prototype flood information inquiry and consultation system, prototype flood forecasting system, etc. In 1994, some of these systems have been put into trial operation and achieved good results. In 1993, flood assessment information system was began to study by China Institute of Geographic Sciences and Natural Resources Research. It was put forward the basic contents of flood, flood remote sensing investigation and monitoring in disaster-stricken areas and five major analysis and calculation models were designed, including a storage capacity estimation model for a good place, flood simulation model range, flood field simulation demonstration model, flood loss assessment and flood risk area[10].

\section{Construction of urban flood prevention and early warning system}

The construction of urban flood prevention and early warning system includes front-end intelligent monitoring network, information transmission, and intelligent management platform(Fig. 1). Based on the requirements of urban flood prevention and early warning construction, water safety monitoring management, auxiliary decision-making, release of flood prevention warning information and long-term supervision, it was developed and realized that business collaboration services, Web services, reporting services, GIS and WebGIS services, application integration and other services. The information and intelligent management of urban flood control and early warning could be realized via systematic integration.

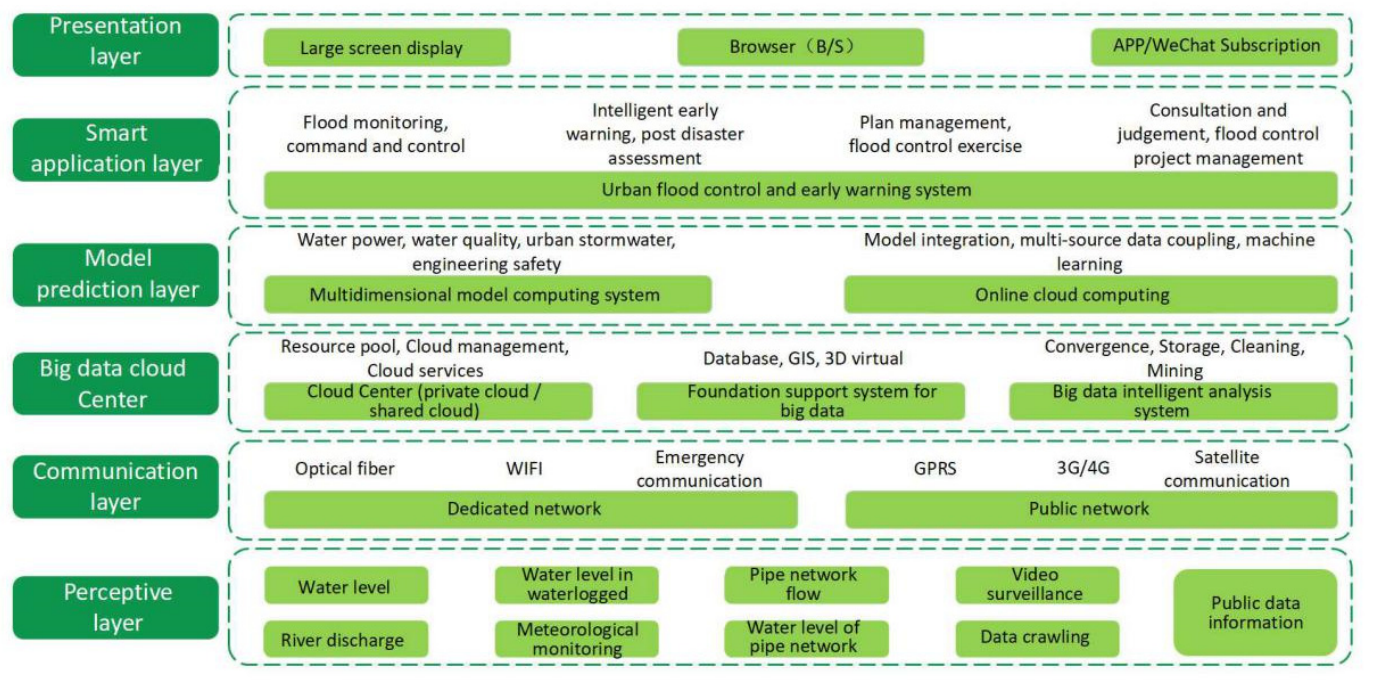

Fig. 1 Platform for urban flood control and early warning information system

(1)Perceptual layer for multi-source data fusion

In the process of constructing urban flood control and early warning system, monitoring of urban flood control should be combined with the data of river water level and water volume, waterlogging-prone area information, meteorological monitoring, pipeline network monitoring, video monitoring and so on. In order to find the hidden relationship between the data and improve the accuracy of the forecast of rainstorm and flood changes, the data were analyzed comprehensively by using multi-source data fusion technology. Unmanned aerial vehicles (UAVs) could be equipped with PIV technology for large-scale flow velocity measurement without interference. Water surface ripple was used as a tracer to improve accuracy of long-range flow velocity measurement and provide data support for urban flood control early warning. The unmanned aerial vehicle based River velocity measurement equipment developed by Beijing Sinfotek Technology Co., Ltd. provides an extremely important supplementary role in monitoring wide river basins, lakes and canals. Obtaining the first-hand frontier data by overlook water surface and conditions of adjacent ground was helpful to timely monitor changes of water environment to make a targeted early warning and take effective action.

(2)Communication layer realizes multi-network transmission

Because monitoring points were distributed in different monitoring scenarios, communication networks need to be designed according to environment and sensor equipment. In order to meet the needs of various data transmission scenarios, fiber-optic networks, wireless 
networks and satellite communications were integrated for long-distance transmission. Transmission equipment could also be compatible with solar panels, power supply, battery and other power supply modes.

(3)Big data cloud center realizes data standardization storage

The automatic online monitoring system improves the data acquisition frequency and makes data volume increase dramatically. Finding the way to get effective data quickly has become the primary problem in the process of big data analysis. Through data interaction verification and correction, the physical location correlation of the perceptual layer intelligent hardware is used to verify the data and realize the real-time error correction and correction of the data. The real-time error correction and correction of data were realized by verifying data of each other based on physical location correlation of perceptual layer intelligent hardware. According to the correlation of large data, trend prediction is made by combining water quality, water level, discharge, current velocity, topographic information, meteorological information and other multi-source data to support decision-making. In order to improve prediction accuracy of model, optimized neural network algorithm was used to automatically calibrate model parameters, so that the computational model could realize autonomous learning. The big data cloud center integrates data security and resource sharing. To ensure data security and unlimited scalability of services, private clouds were used to run core business and public clouds were used to provide external services. Through service customization, intelligent voice service, video surveillance service and image recognition service could be accessed to the system.

(4)Multi-module coupling calculation in the model to improve predictive efficiency

Mathematical models are like human brain to human beings. CCHE, SWMM, DELFT3D and other models were integrated into system. Coupled with other simulation modules, such as surface runoff generation and confluence, pipe network confluence, one-dimensional hydrodynamic and two-dimensional hydrodynamic. For example, the use of drainage module were involved management and calculation of spatial data at multiple levels. The model needed support of GIS technology in GIS. Relying on the GIS platform, the spatial information could be integrated efficiently, so as to achieve pre-processing of spatial data before simulation and post-processing, re-analysis and visualization of results after simulation. Based on the results of remote sensing image recognition and analysis, combined with distribution of pipeline network, pumping station, LID facilities and so on, the grid of blocks were reasonably divided. The infiltration model and confluence model were used to calculate runoff yield of each plot. When entering node of network, the output results of hydraulic model were used to get overflow point and overflow of network. The spillway data were further simulated by a two-dimensional hydrodynamic model to predict the range of water logging, thus improving prediction efficiency.

(5) Smart business applications provide decision making

The city flood prevention and early warning system was limited access and privileged management to users at different levels. Running different business function modules under different business permissions and providing professional and convenient business services in different roles. The main business functions was included flood monitoring, command and dispatch, intelligent early warning, post-disaster assessment, plan management, flood control dispatch drill, consultation and decision, and flood control dispatch management.

(6)Multi-functional display layer provides comprehensive interconnection

Business results publishing and displaying were used various advanced technologies. Specific display methods were included monitoring center (PC side, large screen), consultation center (large screen, workstation) and staff (PC, Weixin, APP). The display terminal communicates with data computing service center was through network to support daily operation and maintenance management of urban flood warning system.

\section{Prospects for the development of urban flood control and early warning system}

In the era of Internet of Things, big data and cloud computing, the scene of urban flood warning decision-making is very different from the past. Urban flood control environment has become very complex, which puts forward many new requirements for the development of urban flood control early warning system.

\subsection{New demand of urban flood control in the era of big data}

With the continuous development of urban construction, urban flood has become increasingly prominent, and has become a constraint factor for the sustainable development of social economy in the region. The construction of urban drainage network can not meet the requirements of rapid expansion of drainage requirements. Improving the ability of monitoring and forecasting rainstorm and flood and emergency management of relevant functional departments is an important problem to be solved urgently.

In the process of urban flood control and early warning system construction, as our country is still in the process of exploration and development, inevitably, there will be some problems, such as:

(1)The monitoring system has not been systematized, the data sources are single

Systematic monitoring is an important means to monitor and control urban stormwater. Because monitoring measures are not systematic and perfect, they can not provide sufficient data support for water resources scheduling. Establish an effective and thorough system monitoring network, and adopt mathematical model to simulate and calculate the urban drainage to 
make an accurate prediction, and provide decision support for urban flood control and water resources allocation.

(2)Lack of multi-dimensional models and long-term data accumulation can not achieve accurate prediction.

Increasing the accuracy of prediction and early warning of mathematical models requires constant accumulation of data.Through the cleaning and excavation of the data, the simulation calculation of the model is used to realize the intelligent functions such as auxiliary decision making, emergency response, and automatic generation of the plan. Long-term data accumulation is a prerequisite for numerical simulation and machine learning.

(3)Lack of data fusion, prone to information islands.

The lack of data interconnection and sharing between departments and between supervisors and subordinates will affect data fusion, easily appear information islands, and affect the improvement of work efficiency, thus hindering the fine management and construction of urban flood control. With the construction and development of smart city, the degree of informatization of water safety management has been gradually raised.

\subsection{Prospect}

(1)Establishment of sensing system

A high-precision urban DEM which can reflect the underlying surface information of complex cities is constructed by using airborne remote sensing stereo image pairs and vehicle-borne laser 3D modeling and measurement system. The DEM is modified by characteristic elements of building boundary, water system and road to meet the needs of urban rainfall and flood analysis.Different from the traditional method of urban rainstorm and flood simulation based on hydro-hydraulics, the method of urban rainstorm and flood process simulation based on spatial model analysis integrates multi-source remote sensing data, rainfall data and urban underlying surface information, and builds a flood disaster case database under complex environmental conditions. On the basis, the new mode of urban flood control and early warning is studied. The probability theory is used to analyze the frequency and possibility of flood occurrence in future scenarios and to warn the flood disaster.

(2)To develop predictive forecasting methods

For prediction and prediction, data can be analyzed and predicted by decision tree. Decision tree is also one of the most classical algorithms in data mining. Decision tree is probability that expected value of net present value is greater than or equal to zero by forming a decision tree on the basis of the known probability of occurrence of various situations. The decision analysis method of evaluating project risk and feasibility judging is a graphic method of intuitive application of probability analysis. It is a method of classifying data based on information theory.

(3)Multi-source data fusion

The standard database system is constructed by multi-source data fusion, and the auxiliary decision-making of urban flood control early warning is promoted by large data. Based on the results of data simulation, a complete urban flood control and early warning decision-making process is completed. In the past, "data driven" was based on data calculation and statistics to complete decision-making. Incomplete sample data was used in traditional simulations, and the relationship patterns between them were known. However, based on multi-source data fusion, almost all the data were used in large data, not a small amount of sample data. The relationship between many data is unknown. Through data cleaning and mining, we can discover the hidden association between the huge data sets and provide decision support.

This paper expounds the development, construction and prospects of urban flood control early warning system, discusses the new requirements for urban flood control early warning system in the new era of information technology development, and analyzes and prospects the combination of cloud computing and large data. It can be concluded that the new generation of information technology will promote the research and development of the next wave of new theories, new models, new technologies and new applications of urban flood control and early warning system.

\section{References}

1. Zhou, H., Liu, J., Gao, C., et al. "Analysis of Current Situation and Problems of Urban Waterlogging Control in China", Journal of Catastrophology, 33( 3):147-151, 2018.

2. Zhang, N.,Q., Huang, H., L., Xu, M., et al. "Flood risk mapping and application in the United Kingdom and its reference to China", China Academic Journal Electronic Publishing House, 28(3):45-47, 2017.

3. Du, M.,Y., Liu, Y. "The Application of Internet of Things in Urban Fine Management", Science of Surveying and Mapping, 42(7):94-101, 2017.

4. Li, Q. "Implementation and Application of Urban Hydrological Flood Warming System", J. Zhejiang Univ of Wat. Res\&Electric Pow., 22(1): 68-71,2017.

5. Di, S.,H., Liu, H., C., Su, H., F., et al. "Current Situation and Challenges of Rainstorm Warning System and Emergency Response Management in Urban Region of Beijing", China Flood and Drought Management, 26(3):49-53, 2016.

6. Wang, L., Liu, H., C., Pan, X., Y., et al. "Construction and Demonstration of Urban Flood Modeling in Beijing", China Flood and Drought Management, 25(4):33-36, 2015.

7. Dong, S., X., “Application of Remote Video Monitor and Control System in Municipal Drainage System", China Water \& Wastewater, 31(9):129-131, 2015.

8. Ye, C., f., Zhang. Y., C., Cheng, W., M., et al. "Analysis on Urban Pluvial Floods and Causes During Rapid Development in Beijing", China 
Flood and Drought Management, 28(2):19-25, 2018.

9. Ding, Z., X., Li, N., "Application of Realtime Flood Risk Analysis in Flood Emergency and Prevention", China Flood and Drought Management, 28(7):1-7, 2018.

10. Yuan, J., “Application Research on Communication Technology in Urban Flood Monitoring and Dispatching System", Instrumentation Technology, 8:38-40, 2014. 